Original research article

\title{
Comparison of biochemical response between the minimally invasive and standard open posterior lumbar interbody fusion
}

\author{
Petr Linzer ${ }^{a, *}$, Michal Filip ${ }^{a}$, Patrik Jurek $^{a}$, Tomáš Šálek ${ }^{b}$, Miroslav Gajdošc ${ }^{c}$ \\ Jiří Jarkouský ${ }^{d}$ \\ ${ }^{a}$ Department of Neurosurgery, Bata Hospital, Zlín, Czech Republic \\ ${ }^{\mathrm{b}}$ Department of Biochemistry, Bata Hospital, Zlín, Czech Republic \\ ${ }^{\mathrm{c}}$ Department of Neurosurgery, Pavol Josef Šafárik University, Košice, Slovakia \\ ${ }^{\mathrm{d}}$ Institute of Biostatistics and Analyses, Masaryk University, Brno, Czech Republic
}

\section{A R T I C L E I N F O}

Article history:

Received 1 June 2015

Accepted 14 October 2015

Available online 6 November 2015

\section{Keywords:}

Creatine kinase

Interleukin-6

Lumbar interbody fusion

Minimally invasive approach

Myoglobin

\begin{abstract}
A B S T R A C T
Background: The mini-invasive open posterior lumbar fusion procedure (mini PLIF) procedure is an alternative to standard open procedure (open PLIF) and is intended to reduce surgery-related trauma. The measuring of suitable biochemical factors enables objective comparison of the invasiveness of spinal surgery procedures.

Methods: Prospectively collected data on myoglobin, creatine kinase, interleukin-6, C-reactive protein levels and intensity of low back pain and radicular pain in one-level mini PLIF and open PLIF procedures were analysed. The mini PLIF and the open PLIF groups included 27 and 23 patients, respectively. The collection of blood samples and clinical data were performed preoperatively and on postoperative days 1, 3 and 7. The non-paired t-test was used for statistical evaluation.

Results: We did not found any statistically significant differences of myoglobin and creatine kinase levels between the groups. In the open PLIF group the IL-6 levels were significantly higher than in the mini PLIF group on postoperative day 3. CRP levels showed significant lower stress response in favour of the mini PLIF group on postoperative days 3 and 7. Levels of post-op low back pain on day 3 were significantly lower in mini PLIF group. Also intensity of radicular pain on day 1 and 3 were lower also mini PLIF group.

Conclusion: The extent of myonecrosis was comparable in both techniques. The analysis of the IL- 6 and CRP levels showed significantly lower systemic inflammatory response in mini PLIF technique. The mini PLIF technique provides transiently lower postoperative pain levels.
\end{abstract}

(C) 2015 Polish Neurological Society. Published by Elsevier Sp. z o.o. All rights reserved.

\footnotetext{
* Corresponding author at: Department of Neurosurgery, Bata Hospital, Havlíčkovonábřeži 600, 76275 Zlín, Czech Republic.

Tel.: +420 603927931; fax: +420 577552107.

E-mail address: petrlinzer@volny.cz (P. Linzer).

http://dx.doi.org/10.1016/j.pjnns.2015.10.008

0028-3843/@ 2015 Polish Neurological Society. Published by Elsevier Sp. z o.o. All rights reserved.
} 


\section{Introduction}

The standard posterior surgical approach for posterior interbody fusion (PLIF) is a widely used surgical technique for treatment of a degenerative disease of the lumbar spine. Nowadays various minimally invasive modifications of open fusion procedures have become more and more popular among spinal surgeons. The aim of mini-invasive surgical techniques is the reduction of undesirable collateral soft tissue damage related to an open approach. Published data comparing open and mini-invasive operational techniques show comparable clinical as well as radiological results [1-4]. Frequently mentioned advantages of mini-invasive techniques are lower blood loss, faster recovery, shorter length of stay and also lower frequency of inflammatory complications [3,5-8]. However, mini-invasive operational techniques are technically demanding, expensive and take longer time. Mastering of the technique also requires a long learning curve. To confirm or refuse the hypothesis that mini-invasive technique is justified and advantageous, objective methods may be employed. An objective comparison of the invasiveness of surgical procedures proposes the biochemically oriented approach. A postoperative monitoring of suitable biochemical markers enables an assessment of the inflammatory response and the extent of muscle damage.

Any surgical intervention induces stress and an inflammatory response in the body, which is proportional to the access size, blood loss and length of exposure $[9,10]$. Proinflammatory interleukin-6 (IL-6) is produced at the site of the insult and subsequently enters the peripheral blood. Liver synthesis of Creactive protein (CRP) is induced in response to the release of interleukin-1 (IL-1) and IL-6. Many authors comparing invasiveness of the surgical procedures studied the levels of the mentioned biochemical factors [11-16]. Analogously to their use in the diagnosis of myocardial ischaemia, elevated levels of specific muscle proteins reflect the size of the lesion of the skeletal muscles. An insult leads to an increase in serum concentrations of a number of proteins, such as creatine kinase (CK), myoglobin (MYO), lactate dehydrogenase, aminotransferases and others [14,15,17-22].

The objective of this study is to compare the invasiveness between the minimally invasive PLIF (mini PLIF) and the standard open PLIF (open PLIF) based on total stress response (IL-6, CRP) and muscle trauma (CK and MYO).

\section{Material and methods}

We analysed prospectively collected data from patients operated on using the one-level mini PLIF technique and the open PLIF. 27 consecutive patients were included in the mini PLIF group and 23 patients underwent the open PLIF procedure in the same period. Inclusion criteria were: lumbar degenerative disc disease, radiological and/or clinical signs of segmental instability, clinical signs of spinal nerve compression and 6 months of unsuccessful conservative treatment. Exclusion criteria were previous lumbar spine procedures, corticosteroid medication, abnormal baseline preoperative values of the observed parameters and patients with infectious, hepatic, cardiac and autoimmune diseases. Patients with suspected postoperative inflammatory complications and intramuscular medication delivery were also excluded. The choice of surgical technique was left to the individual preferences of the participating neurosurgeons. Procedures were performed under general anaesthesia and perioperative administration of anaesthetics included sevoflurane, intravenous application of propofol $2.5 \mathrm{mg} / \mathrm{kg}$, sufentanil $0.2-0.4 \mu \mathrm{g} / \mathrm{kg}$ and cisatracurium $0.15 \mathrm{mg} / \mathrm{kg}$.

The mini PLIF surgical technique included unilateral decompression and posterior interbody fusion supplemented by percutaneous pedicular fixation as described by Logroscino et al. [23]. The procedure was performed in a prone position with an appropriate padding of a patient to avoid abdomen compression. After X-ray localisation a short paramedial incision on the side of prevailing symptoms, the dilatation and insertion of a $21 \mathrm{~mm}$ wide tubular retractor were performed. Decompression, discectomy and preparation of interbody space were performed under control of microscope. One cage filled with autologous bone was inserted into interbody space (Capstone - Medtronic Sofamor Danek USA, Inc.; Concorde - DePuy Synthes, USA). Bone material was harvested from laminae during an approach into the spinal canal. After cage insertion and wound closure, percutaneous bilateral insertion of a pedicle screw fixation followed (CD Horizon Sextant II - Medtronic Sofamor Danek USA, Inc., VIPER 2 - DePuy Synthes, USA).

In the open PLIF group the surgical technique included a midline incision, soft tissue dissection, paravertebral muscle dissection and retraction up to bases of transverse processes. After a standard laminectomy and bilateral discectomy one or two interbody cages were inserted. The insertion of a pedicle screw fixation followed (XIA - Stryker, USA).

Samples for biochemical analysis were collected using the standard venipuncture technique on the day before surgery, and on postoperative days 1, 3 and 7. Samples were sent to the laboratory and measured in an automatic analyser. CRP levels were measured using a high-sensitive latex immunoturbidimetric assay on the Abbott Architect analyser. IL- 6 levels were determined using a sandwich immunoassay with electrochemiluminescence detection on the Cobas e-411 analyser. CK activity was measured using the enzymatic photometric method on the Abbott Architect analyser, while myoglobin levels were determined by a particle enhanced immunoturbidimetric assay on the same analyser. The normal reference laboratory values were as follows: CRP $\leq 5 \mathrm{mg} / \mathrm{L}$, IL- $6 \leq 7 \mathrm{ng} / \mathrm{L}$, $\mathrm{CK} \leq 3.25 \mu \mathrm{kat} / \mathrm{L}$ and myoglobin $\leq 117 \mu \mathrm{g} / \mathrm{L}$. Reference ranges were established according to manufacturer and the laboratory procedures performed according to International Federation of Clinical Chemistry and Laboratory Medicine (IFCC) recommendation.

Preoperative and post-op values on the 10-grade visual analogue pain scale for low back pain (VAS BP) and radicular pain (VAS R) were also recorded.

Any intramuscular administration of the medications was excluded during the preoperative and postoperative periods. Patients were allowed to ambulate on the third day after the surgery. The time of operation was obtained from the clinical records. Postoperative analgesia included patient-controlled administration of paracetamol, metamizol, and tramadol. 
Standard descriptive statistics were used for the data description; absolute and relative frequency for categorical data and a mean supplemented by $95 \%$ confidence interval (CI) for continuous data. Statistical significance of the differences between the types of surgeries was assessed by Student's t-test for two independent samples. A relationship between continuous variables was assessed using a Spearman correlation coefficient. In all tests the value $\alpha=0,05$ was adopted as a border of statistical significance.

The study was approved by the local Ethics Committee and all patients signed an informed consent form.

\section{Results}

Demographic characteristics, time of surgical procedure and operated levels are shown in Table 1 . No patient required a blood transfusion in the postoperative period.

\subsection{Changes in muscle proteins}

Serum myoglobin levels reached their peak on the first postoperative day. Compared to the preoperative values the difference in the serum levels in both groups was statistically significant on postoperative days 1 and $3(p<0.001)$. On postoperative day 1 the MYO level in the mini PLIF group (369.2 $\mu \mathrm{g} / \mathrm{L}, 95 \%$ CI: $255.4 ; 483.0$ ) was lower compared to the open PLIF group $(464.4 \mu \mathrm{g} / \mathrm{L}, 95 \% \mathrm{CI}: 338.7 ; 590.1)$, but the difference was not statistically significant (Fig. 1). Insignificant lower values in the mini PLIF group $(71.6 \mu \mathrm{g} / \mathrm{L}, 95 \% \mathrm{CI}: 43.1$; 100.1) than in the open PLIF $(90.1 \mu \mathrm{g} / \mathrm{L}, 95 \% \mathrm{CI}: 53.9 ; 126.4)$ persisted on postoperative day 3. On postoperative day 7 , myoglobin values in both groups did not reach statistically significant differences compared to the preoperative levels $(p=0.235)$.

Operational procedures induced a statistically significant elevation of CK levels on postoperative days 1 and 3 in both groups. The highest levels were observed on the first postoperative day. On day 1 mean CK levels in the mini PLIF and the open PLIF groups were $24.5 \mu \mathrm{kat} / \mathrm{L}$ (95\% CI: 19.3, 29.7)
Table 1 - Patient demographic data (mean values supplemented by $95 \%$ CI, differences tested by t-test).

\begin{tabular}{|c|c|c|c|c|}
\hline & Total & Mini PLIF & Open PLIF & $p$ \\
\hline $\begin{array}{l}\text { No. of } \\
\text { patients }\end{array}$ & 50 & 27 & 23 & \\
\hline Age & $\begin{array}{l}51.2 \\
(48.9-53.6)\end{array}$ & $\begin{array}{l}49.9 \\
(47.1-52.6)\end{array}$ & $\begin{array}{l}52.9 \\
(49.0-56.7)\end{array}$ & 0.206 \\
\hline $\begin{array}{c}\text { Gender } \\
(\mathrm{M} / \mathrm{F})\end{array}$ & $25 / 25$ & $13 / 14$ & $12 / 11$ & 1.000 \\
\hline BMI & $\begin{array}{l}27.8 \\
(26.8-28.8)\end{array}$ & $\begin{array}{l}26.9 \\
(25.7-28.1)\end{array}$ & $\begin{array}{l}28.8 \\
(27.2-30.4)\end{array}$ & 0.057 \\
\hline $\begin{array}{l}\text { Time for } \\
\text { operation } \\
\text { (min) }\end{array}$ & $\begin{array}{l}144.9 \\
(138.9-151.0)\end{array}$ & $\begin{array}{l}148.2 \\
(140.6-155.7)\end{array}$ & $\begin{array}{l}141.1 \\
(131.4-150.7)\end{array}$ & 0.255 \\
\hline \multicolumn{5}{|c|}{ Level of fusion (no. of pts/\%) } \\
\hline L2/L3 & $2(4 \%)$ & $1(3.7 \%)$ & $1(4.3 \%)$ & 1.000 \\
\hline L3/L4 & $2(4 \%)$ & $1(3.7 \%)$ & $1(4.3 \%)$ & 1.000 \\
\hline L4/L5 & $38(76 \%)$ & $21(77 \%)$ & $17(73.9 \%)$ & 0.309 \\
\hline L5/S1 & $8(16 \%)$ & $4(14.8 \%)$ & $4(17.4 \%)$ & 1.000 \\
\hline
\end{tabular}

and $22.4 \mu \mathrm{kat} / \mathrm{L}$ (95\% CI: 17.6, 27.3), respectively. On postoperative day 3 values of CK decreased to $21.3 \mu \mathrm{kat} / \mathrm{L}$ (95\% CI: 15.5; 27.0) and $18.4 \mu \mathrm{kat} / \mathrm{L}$ (95\% CI: 12.4; 24.4). The differences were not statistically significant, however (Fig. 2). On postoperative day 7 levels of CK were the same $(3.5 \mu \mathrm{kat} / \mathrm{L})$.

\subsection{Markers of inflammatory response}

Mean IL-6 levels reached their peak on the first postoperative day in all groups but when compared to the preoperative levels, the difference was significant on all postoperative analysis $(p<0.001)$. The mean IL-6 levels on postoperative day 1 were $57.9 \mathrm{ng} / \mathrm{L}(95 \%$ CI: 43.1; 72.8) in the mini PLIF group and $82.5 \mathrm{ng} / \mathrm{L}$ (95\% CI: 55.4 ; 109.7) in the open PLIF group. This difference was not statistically significant. On day 3 levels of IL-6 dropped to $13.6 \mathrm{ng} / \mathrm{L}(95 \%$ CI: $10.8 ; 16.3)$ in the mini PLIF and to $35.5 \mathrm{ng} / \mathrm{L}(95 \% \mathrm{CI}: 28.4 ; 42.7)$ but on this day the difference between groups were highly significant (Fig. 3).

Both in the mini PLIF and the open PLIF groups the elevations of CRP levels were statistically significant on all postoperative days under investigation and reached their peak

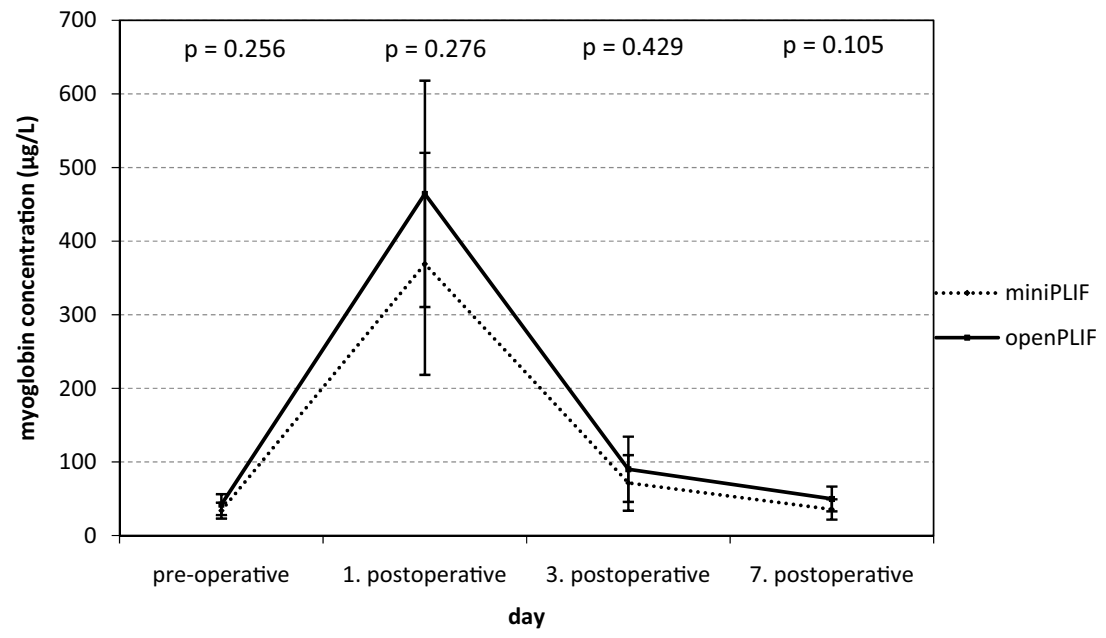

Fig. 1 - Mean and standard deviations of MYO levels on postoperative days 1, 3 and 7 ( $p$-value of student t-test for two independent samples). 


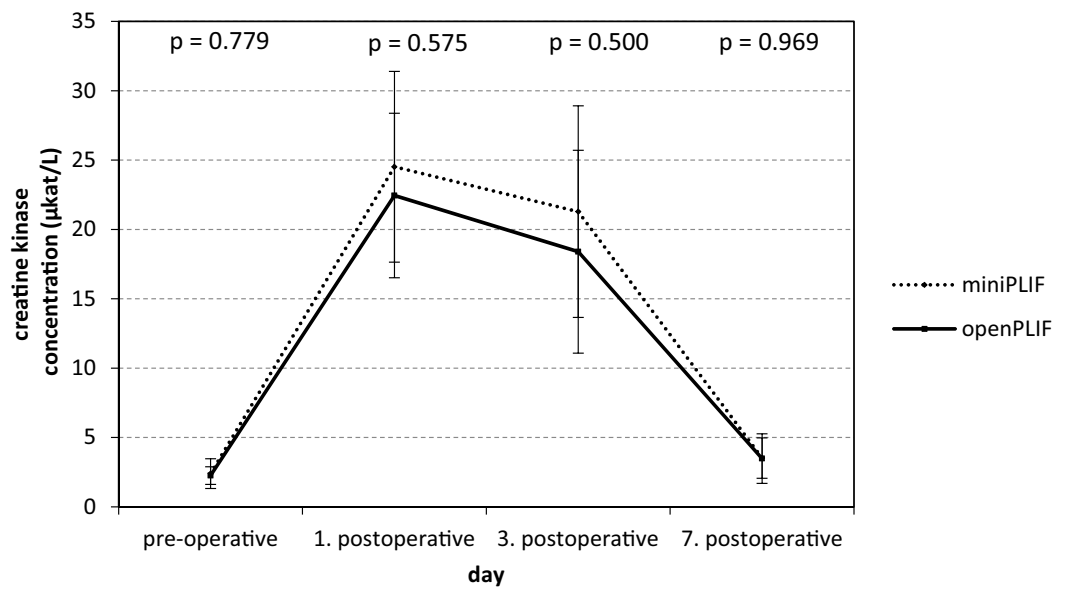

Fig. 2 - Mean and standard deviations of CK levels on postoperative days 1, 3 and 7 ( $p$-value of student t-test for two independent samples).

on the 3rd postoperative day $(p<0$.001). On postoperative days 1,3 and 7 mean CRP levels in the mini PLIF group reached $68.4 \mathrm{mg} / \mathrm{L}$ (95\% CI: 55.6; 81.2), $99.0 \mathrm{mg} / \mathrm{L}$ (95\% CI: 79.0; 118.9) and $17.4 \mathrm{mg} / \mathrm{L}$ (95\% CI: 11.7; 23.0), respectively. The corresponding values in the open PLIF group were $72.7 \mathrm{mg} / \mathrm{L}$ (95\% CI: 53.7 ; 91.6), $140.8 \mathrm{mg} / \mathrm{L}(95 \% \mathrm{CI}: 115.5 ; 166.0)$ and $27.3 \mathrm{mg} / \mathrm{L}(95 \% \mathrm{CI}$ : 19.9; 34.8). On days 3 and 7 the differences between groups were statistically significant in favour of the mini PLIF group (Fig. 4).

We also performed a sub-analysis of the effect of the patients' age on biochemical markers' levels (non-parametric Spearman correlation). In the case of muscle-related parameters (CK, MYO), no moderate $(0.3<r<0.6)$ or strong $(r>0.6)$ correlation were recorded. For stress markers, a moderately significant correlation was observed for IL-6 on postoperative day $1(r=0.350, p=0.013)$. On day 7 the correlation was significant to a low extent $(r=0.208$, $p=0.049$ ). The duration of the surgical procedure was another analysed factor. We did not observe any moderate or strong correlation.

\subsection{Changes in VAS BP and VAS $R$}

Preoperative mean VAS BP values were 5.02 in the open PLIF group and 4.72 in the mini PLIF group. The difference was not significant $(p=0.61)$. Post-op mean values in the open PLIF group on day 1, 3 and 7 were 6.33, 5.4 and 3.3. In the mini PLIF group corresponding values were 5.28, 3.9 and 2.5. The VAS BP values were significantly lower in the mini PLIF group on the post-op day 3 (Fig. 5).

The statistical analysis of the relationship between the VAS $B P$ values and muscle proteins' levels in the open PLIF and mini PLIF groups did not prove any moderate or strong correlation. The analysis of stress markers in the mini PLIF group shoved moderate correlation between CRP level on post-op day 3 and VAS BP values on post-op. days 3 and $7(r=0.429, p=0.014$, respectively $r=0.59, p=0.001$ ). In the open PLIF group no statistically significant correlations between VAS BP and levels of analysed biochemical factors were found.

Preoperative mean VAS R values were 5.86 in the open PLIF group and 5.18 in the mini PLIF group. Differences were

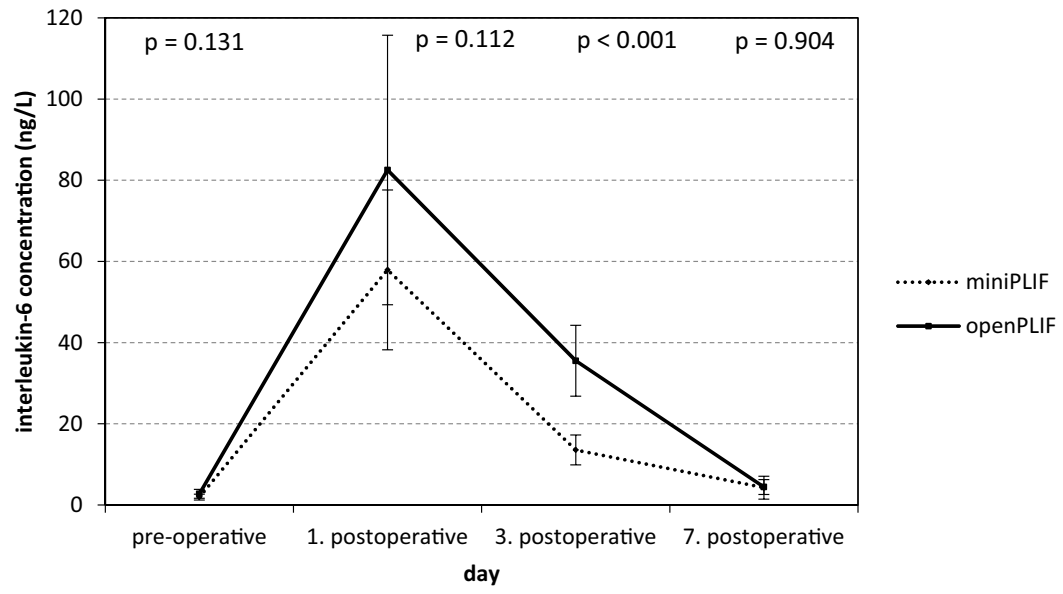

Fig. 3 - Mean and standard deviations of IL-6 levels on postoperative days 1, 3 and 7 ( $p$-value of student t-test for two independent samples). 


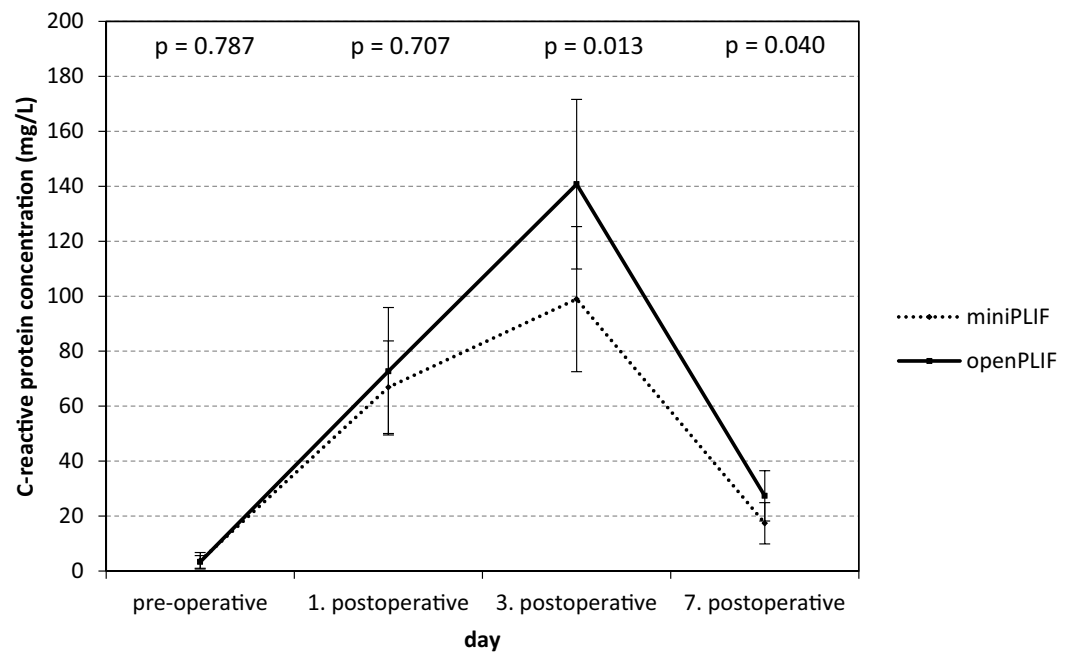

Fig. 4 - Mean and standard deviations of CRP levels on postoperative days 1, 3 and 7 ( $p$-value of student t-test for two independent samples).

insignificant $(p=0.35)$. Values on post-op days 1,3 and 7 were 3.6, 3.75 and 1.7 in the open PLIF group and 2.22, 1.85 and 1.5 in the mini PLIF group. On post-op days 1 and 3 significantly lower values in the mini PLIF group were found (Fig. 6).

Also the relationship between VAS R and muscle proteins' levels did not reveal any significant correlation. The analysis of relationship between stress markers and VAS R values in the mini PLIF group shoves the moderate correlation on the day 7 and IL-6 levels on day 1 and $7(r=0.419, p=0.029$, respectively $r=0.461, p=0.016)$. In case of CRP levels the moderate correlation between CRP level on the day 3 and VAS R was found. In the open PLIF group the only correlation was between the CRP level on the day 1 and VAS R value on the day 7 $(r=0.389, p=0.089)$.

\section{Discussion}

An extensive dissection of soft tissues and paravertebral muscles is an inevitable part of dorsal surgical approaches used to perform posterior interbody fusion. Dissection, dilatation and retraction of the paraspinal muscles lead to myonecrosis, denervation and subsequent postoperative muscle atrophy and scarring [24-30]. Increased intramuscular pressure and ischaemia have been reported to play a role in the pathophysiology of this condition [31-33]. Damage to the paraspinal muscles, and in particular multifidi muscles due to their medial localisation, can contribute to the development of segmental instability. Paraspinal muscle damage has been associated with poor outcome after spine surgery procedures in the lumbar spine area $[27,28]$.

Monitoring of serum CK levels as a marker of myonecrosis caused by necrosis of the paraspinal muscles was used in a number of studies dealing with muscle trauma in association with dorsal spinal surgery procedures [14,15,18,34,35]. This methodology is based on the assumption that the dorsal spine surgery interventions induce detectable elevation of CK levels $[36,37]$. Kumbhare et al. demonstrated a good correlation between the CK levels and muscle area during spine surgery intervention [38]. A significant relationship has been also

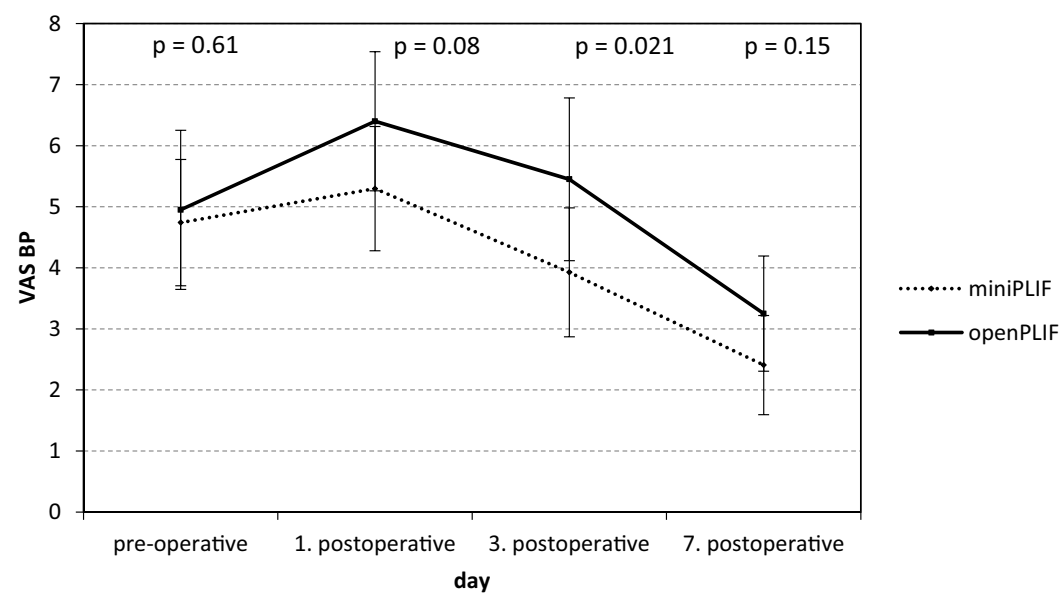

Fig. 5 - Mean and standard deviations of VAS BP values on postoperative days 1, 3 and 7 ( $p$-value of student t-test for two independent samples). 


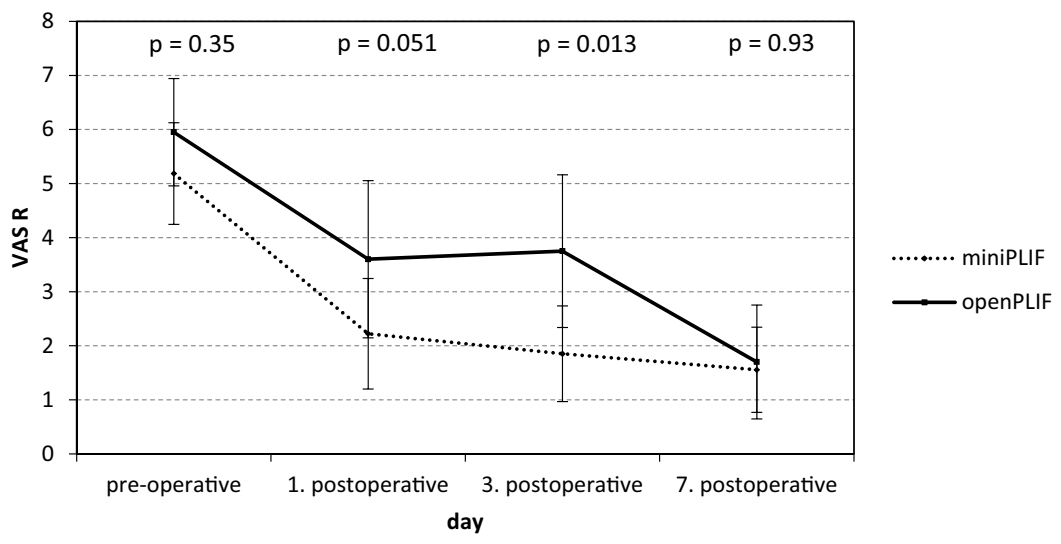

Fig. 6 - Mean and standard deviations of VAS R values on postoperative days 1, 3 and 7 ( $p$-value of student t-test for two independent samples).

reported between the $\mathrm{CK}$ levels and the invasiveness of the surgery $[17,22]$.

Based on the results of the CK levels in our study we were not able to prove the protective effect of the mini PLIF technique on paravertebral muscles. The lower CK levels in open PLIF group were somewhat surprising. On the contrary, the observed MYO levels showed more favourable results for the mini PLIF technique. The differences of the MYO levels between studied groups were also not significant and did not prove superiority of any of the studied techniques.

Studies assessing the muscle damage of the open and the minimally invasive PLIF or TLIF procedures showed inconsistent results. The study comparing CK levels in 20 patients operated on using the open and the minimally invasive PLIF techniques showed significantly lower levels in the mini PLIF group [14]. Unexpectedly higher levels in the minimally invasive TLIF technique compared to the open procedure were detected in the study of Adogwa et al. [1]. The reasons for those differences are not clear, possibly the variability in $\mathrm{CK}$ concentrations may be responsible for it [35]. As proposed by Adogwa, the explanation for this finding may be "iatrogenic compartment" syndrome that develops during dilatation and tubular retractor placement in a contained surgical bed [1].

The cause of ascertained differences in results between $C K$ and MYO may reside in the different way that CK and MYO molecules reach the circulation. While CK is predominantly transferred into the serum through the lymphatic circulation, MYO is released directly into the circulation following myonecrosis and is known to achieve peak concentrations earlier [39]. It is questionable as to which kind of used muscle marker is more convenient for detecting the extent of muscle damage. Further research is needed to answer the question.

Although the protective effect of the mini-invasive surgical technique was not proved, it is worth to mention, that other factors such as possible denervation effect due to muscle detachment, lateral displacement and dorsal ramus of spinal nerve injury were not investigated.

The comparison of the invasiveness of different surgical procedures is based on the correlation between peak IL-6 and CRP concentrations and the extent of surgical trauma. Level of
IL-6 after insult rises rapidly and peaks on postoperative day 1 [10]. Unlike muscle factors, published data on IL-6 after surgical procedures and our findings correspond well. Significantly lower IL-6 levels for the mini PLIF operation on postoperative day 1 and 3 were observed in the study of Kim et al. [14]. Our results show lower inflammatory response in the mini PLIF group likewise, but differences are statistically significant on postoperative day 3 only. For CRP, the maximum level was recorded on postoperative day 3 in both groups. This latency compared to the IL- 6 levels can be explained by its liver synthesis. Similar observations were also made in other studies $[12,40]$. Analysis of CRP levels proved statistically significant lower values in the mini PLIF group on postoperative days 3 and 7. This finding confirms lower operational stress in the mini PLIF group and agrees with IL- 6 analysis as well. We did not analyse IL 6 and CRP levels beyond postoperative day 7. According to published studies, normalisation of CRP levels can be expected by day 14 after the procedure [16,40].

Considering the relation between the time for the operation and postoperative trunk muscle performance or postoperative improvement, we expected higher levels of muscle factors in longer operations [24,31]. Surprisingly, we have not found any correlation between the time for operation and the degree of muscle damage. Given the reported atrophy of type II muscle fibres with increasing age, we expected lower CK levels in the elderly [30]. In our study, we have not found any effect of the age on blood CK and MYO levels. This finding is in agreement with the study published by Arts et al. [17]. Based on the IL- 6 and CRP values, it can be concluded that elderly patients have a more pronounced stress response on the first postoperative day.

Comparison of VAS BP values favours the mini PLIF method, but statistically significant difference was proved on post-op day 3 only. Final values on the post-op day 7 showed comparable results. The VAS BP values correspond with IL- 6 and CRP levels well and implies the possible relationship between clinical values and levels of proinflammatory factors. The correlation analysis of muscle damage markers did not revealed any relationship to early post-op clinical results and its clinical relevancy remains unclear. 
In spite of the fact that the values in the mini PLIF group shoved faster decrease of VAS R values, the final results proved comparable efficacy in reducing radicular pain. Analysis of the relationship between stress markers and the VAS R levels in the mini PLIF group shoved possible connection between stress markers and radicular pain level. In agreement with previous findings concerning VAS BP, we did not prove any relationship of post-op VAS R and analysed muscle factors.

\section{Conclusion}

The analysis of the biochemical stress response of mini-invasive and open PLIF techniques showed significantly lower systemic inflammatory response in mini-invasive PLIF technique. The analysis of creatine kinase and myoglobin levels showed the comparable extent of myonecrosis in both techniques.

\section{Conflict of interest}

None declared.

\section{Acknowledgement and financial support}

None declared.

\section{Ethics}

The work described in this article has been carried out in accordance with The Code of Ethics of the World Medical Association (Declaration of Helsinki) for experiments involving humans; Uniform Requirements for manuscripts submitted to Biomedical journals.

\section{R E F E R E N C E S}

[1] Adogwa O, Kwame J, Min ET, Isaar N, Carr K, Huang K, et al. Extent of intraoperative muscle dissection does not affect long-term outcomes after minimally invasive surgery versus open-transforaminal lumbar interbory fusion surgery: a prospective longitudinal cohort study. Surg Neurol Int 2012;3(Suppl. 5):S355-61.

[2] Dhall SS, Wang MY, Mummaneni PV. Clinical and radiographic comparison on mini-open transforaminal lumbar interbody fusion with open transforaminal interbody fusion in 42 patients with long-term follow-up. J Neurosurg Spine 2008;9(6):560-5.

[3] Isaacs RE, Podichetty VK, Santiago P, Sandhu FA, Spears J, Kelly K, et al. Minimally invasive microendoscopy-assisted transforaminal lumbar interbody fusion with instrumentation. J Neurosurg Spine 2005;3(2):98-105.

[4] Ntoukas V, Müller A. Minimally invasive approach versus traditional open approach for one level posterior lumbar interbody fusion. Minim Invasive Neurosurg 2010;53(1):21-4.

[5] Kasis AG, Marschman LA, Krishna M, Bhatia CK. Significantly improved outcomes with a less invasive posterior lumbar interbody fusion incorporating total facetectomy. Spine 2009;34(6):572-7.
[6] O'Toole JE, Eichholz KM, Fessler RG. Surgical site infection rates after minimally invasive spinal surgery. J Neurosurg Spine 2009;11(4):471-6.

[7] Park Y, Ha JW. Comparison of one-level posterior lumbar interbody fusion performed with minimally invasive approach or a traditional open approach. Spine 2007;32 (5):537-43.

[8] Parker SL, Lerner J, McGirt MJ. Effect of minimally invasive technique on return to work and narcotic use following transforaminal lumbar inter-body fusion: a rewiev. Prof Case Manag 2012;17(5):229-35.

[9] Baigrie RJ, Lamont PM, Kwiatkowski D, Dallman MJ, Morris PJ. Systemic cytokine response after major surgery. Br J Surg 1992;79(8):757-60.

[10] Sakamoto K, Arakawa H, Mita S, Ishiko T, Ikei S, Egami H, et al. Elevation of circulating interleukin 6 after surgery: factors influencing the serum level. Cytokine 1994;6(2): 181-6.

[11] Al-Jabi Y, El-Shawarby A. Value of C-reactive protein after neurosurgery: a prospective study. Br J Neurosurg 2010;24 (6):653-9.

[12] Houten JK, Tandon A. Comparison of postoperative values for C-reactive protein in minimally invasive and open lumbar spinal fusion surgery. Surg Neurol Int 2011;2:94.

[13] Huang TJ, Hsu RW, Li YY, Cheng CC. Less systemic cytokine response in patients following microendoscopic versus open lumbar discectomy. J Orthop Res 2005;23(2):406-11.

[14] Kim KT, Lee SH, Suk KS, Bae SC. The quantitative analysis of tissue injury markers after mini-open lumbar fusion. Spine 2006;31(6):712-6.

[15] Kim K, Isu T, Sugawara A, Matsumoto R, Isobe M. Comparison of the effect of 3 different approaches to the lumbar spinal canal on the postoperative paraspinal muscle damage. Surg Neurol 2008;69(2):109-13.

[16] Thelander U, Larsson S. Quantitation of C-reactive protein levels and erythrocyte sedimentation rate after spinal surgery. Spine 1992;17(4):400-4.

[17] Arts MP, Nieborg A, Brand R, Peul WC. Serum creatine phosphokinase as an indicator of muscle injury after various spinal and nonspinal surgical procedures. J Neurosurg Spine 2007;7:282-6.

[18] Cohen RG, Katz JA, Skrepnik NV. The relationship between skeletal muscle serum markers and primary THA: a pilot study. Clin Orthop Relat Res 2009;467(7):1747-52.

[19] Dahl B, Schiødt FV, Gehrchen PM, Ramlau J, Kiaer T, Ott P. Gc-globulin in an acute phase reactant and an indicator of muscle injury after spinal surgery. Inflammation research 2001;50(1):039-43.

[20] Martinez-Amat A, Boulaiz H, Prados J, Marchal JA, Padial Puche P, Caba O, et al. Release of aktin into serum after skeletal damage. Br J Sports Med 2005;39(11):830-4.

[21] Poli D, Gemma M, Cozzi S, Lugani D, Germagnoli L, Beretta L. Muscle enzyme elevation after elective neurosurgery. Eur J Anaesthesiol 2007;24(6):551-5.

[22] Suwa H, Hanakita J, Ohshita N, Gotoh K, Matsuoka N, Morizane A. Postoperative changes in paraspinal muscle thickness after various lumbar back surgery procedures. Neurol Med Chir (Tokyo) 2000;40(3):151-5.

[23] Logroscino CA, Proietti L, Pola E, Scaramuzzo L, Tamburrelli FC. A minimally invasive posterior lumbar interbody fusion for degenerative lumbar spine instabilities. Eur Spine J 2011;20(Suppl. 1):S41-5.

[24] Gejo R, Matsui H, Kawaguchi Y, Ishihara H, Tsuji H. Serial changes in trunk muscle performance after posterior lumbar surgery. Spine 1999;24(10):1023-8.

[25] Kawaguchi Y, Matsui H, Tsuji H. Back muscle injury after posterior lumbar spine surgery. Part 2: Histological and histochemical analyses in humans. Spine 1994;19(22): 2598-602. 
[26] Kotil K, Tunckale T, Tatar Z, Koldas M, Kural A, Bilge T. Serum creatine phosphokinase activity and histological changes in the multifidus muscle: a prospective randomized controlled comparative study of discectomy with and without retraction. J Neurosurg Spine 2007;6(2):121-5.

[27] Rantanen J, Hurme M, Falck B, Alaranta H, Nykvist F, Lehto $\mathrm{M}$, et al. The lumbar multifidus muscle five years after surgery for a lumbar intervertebral disc herniation. Spine 1993;18(5):568-74.

[28] Sihvonen T, Hrno A, Paljärvi L, Airaksinen O, Partanen J, Tapaninaho A. Local denervation atrophy of paraspinal muscles in postoperative failed back surgery syndrome. Spine 1993;18(5):575-81.

[29] Taylor H, McGregor AH, Medhi-Zadeh S, Richards S, Kahn N, Zadeh JA, et al. The impact of self-retaining retractors on the paraspinal muscles during posterior spinal surgery. Spine 2002;27(24):2758-62.

[30] Weber BR, Grob D, Dvorak J, Müntener M. Posterior surgical approach to the lumbar spine and its effect on the multifidus muscle. Spine 1997;22(15):1765-72.

[31] Datta G, Gnanalingham KK, Peterson D, Mendoza N, O'Neill $\mathrm{K}$, Van Dellen J, et al. Back pain and disability after lumbar laminectomy: Is there a relationship to muscle retraction? Neurosurgery 2004;54(6):1413-20.

[32] Gille O, Jolivet E, Dousset V, Degrise C, Obeid I, Vital JM, et al. Erector spinae muscle changes on magnetic resonance imaging following lumbar surgery through a posterior approach. Spine 2007;32(11):1236-41.
[33] Kawaguchi Y, Yabuki S, Styf J, Olmarker K, Rydevik B, Matsui $\mathrm{H}$, et al. Back muscle injury after posterior lumbar spine surgery. Spine 1996;21(22):2683-8.

[34] Kirwan JP, Clarkson PM, Graves JE, Lichtfield PL, Byrnes WC. Levels of serum creatine kinase and myoglobin in women after two isometric exercise conditions. Eur J Appl Physiol Occup Physiol 1986;55(3):330-3.

[35] Sayers SP, Clarkson PM. Short-term immobilization after eccentric exercise. Part II: creatine kinase and myoglobin. Med Sci Sport Exerc 2003;35(5):762-8.

[36] Kawaguchi Y, Matsui H, Tsuji H. Changes in serum creatine phosphokinase MM isoenzyme after lumbar spine surgery. Spine 1997;22(9):1018-23.

[37] Kumbhare D, Parkinson W, Dunlop B, Ryan E, Denkers M, Shah AA, et al. Biochemical measurement of muscle injury created by lumbar surgery. Clin Invest Med 2007;30 (1):12-20.

[38] Kumbhare D, Parkinson W, Dunlop B. Validity of serum creatine kinase as a measure of muscle injury produced by lumbar surgery. J Spinal Disord Tech 2008;21(1): 49-54.

[39] Mair J. Tissue release of cardiac markers: from physiology to clinical application. Clin Chem Lab Med 1999;37(11/12): 1077-84.

[40] Aono H, Ohwada T, Kaneko N, Fuji T, Iwasaki M. The postoperative changes in the level of inflammatory markers after posterior lumbar interbody fusion. J Bone Joint Surg (Br) 2007;89-B:1478-81. 